\title{
EXPERIMENTAL RESEARCH RELATING TO THE MANUFACTURE OF PARTS BY THE ADDITION OF MATERIAL
}

\author{
Dana Rizescu ${ }^{1}$, Ciprian Ion Rizescu ${ }^{2}$ \\ 1,2 University POLITEHNICA of Bucharest, Department of Mechatronics and Precision \\ Engineering \\ Splaiul Independenței, nr. 313, postal code 060042, sector 1, Bucharest, ROMANIA \\ dana.rizescu@upb.ro,ciprian.rizescu@upb.ro
}

\begin{abstract}
This work was developed at University POLITEHNICA of Bucharest and there were several challenges regarding technological aspects. Finally, we have succeeded in designing and manufacturing a printed object, according to required conditions. In the paper is presented as compared the manufacturing process by adding the material which uses the raw material in the solid state between two systems to print provided with a extruder and with two extruders. Thus, there were designed and printed a couple of physical models used in sporting activities, in accordance with the 3D models. There are presented some remarks about the quality for the two printing systems.
\end{abstract}

Keywords: 3D Printing, Simulation, Material Adding, Extruder.

\section{Introduction}

As it is known, 3D printing, also called additive manufacturing, this process involves a 3D printer with XYZ axis which creates a 3-dimensional solid object from a digital model by laying down successive layers of material in different shapes.

- Builds the object by deposition of material rather than by removal of material.

- Printing mediums range from paper, plastic, powders, resins, metals.

- Depending on our model, one will want a certain printer type and medium.

- Two Main Types of Printers (among many)

- Fused Deposition Modeling (FDM)

- Selective Laser Melting (SLM)

Generating . STL files

STL - Standard Tessellation Language. This file format is widely used in rapid prototyping and also .STL files are common files used in 3D printing prototype.STL files describe only the surface geometry of a three dimensional object without any representation of colour, texture or other common CAD model attributes.

The .STL format specifies both ASCII and binary representations. Binary files are more com-mon, since they are more compact. An.STL file consists of a list of facet data. Each facet is uniquely identified by a unit normal (a line perpendicular to the triangle and with a length of 1.0) and by three vertices (corners).
The normal and each vertex are specified by three coordinates each, so there is a total of 12 numbers stored for each facet.

CAD design with Inventor. Inventor is one of the world's most used pieces of 3D mechanical CAD design software for creating 3D digital prototypes used in the de-sign, visualization and simulation of products. It uses a parametric solid modelling techniques, that makes it very suitable for the design process of engineered mechanisms and geometriclike exterior shapes, allowing for quick changes and adaptation of the geometric characteristics through a very intuitive workflow. This is the reason we used Inventor 2016 for workpiece design.

To perform a print, the machine reads the design from 3D printable file (.stl file) and lays down successive layers of liquid, powder or sheet material to build the model from a series of cross section.

These layers, which correspond to the virtual cross section from the CAD model, are joined or automatically fused to create the final shape.

The project propose the development of a technology having as result the individualized pieces, in order to fulfill at the same time functional and optional requirements requested by the users. This individualization is achieved by manufacturing of a piece adapted to:

- configuration and special appearance for the customer (shapes, colours, materials, name of the owner, etc.), [1];

- the positioning of the piece in the assembly.

- possibility to change the pieces. 


\section{Camera Base Design}

The steps to be follow for the achievement of the helmet camera base adapted to the user's requirements are listed below. Here are some remarks about how one can developed the design of the camera base (camera support). One of them is to use a scanner for determine the geometric shape of the helmet. Other is the determinethe radius of curvature by measuring it.

The idea of using the information about acquired 3D form of the specific helmet to asses the virtual image of the user arise from two parameters: shapes and colors. There are many shapes of helmets, according to their destination: bike helmet, skate-board helmet, rollers helmet, ski helmet, etc.

These helmets are manufactured of various colors and this is reason the camera base must fit with helmet shape and color.

In figure 1 is presented a ski helmet, without action camera. The design of the camera base is presented in figure 2, as a 3D representation. The name of the owner is written embossed or engraved manner.

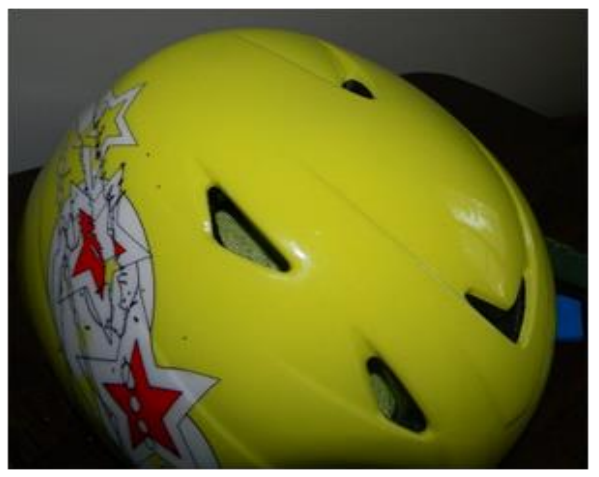

Figure 1: The helmet without printed camera base
Our goal is to go further and integrate the camera base realization in a complete process. Advantages of 3D printing technology are:

- The main advantage of the proposed technology, compared with the classical one, is a better fit to the user's needs;

- The unique design of the camera base ;

- A complete fit to various helmet shapes.

\section{3D Printing}

In the frame of this project there were used two 3D printers, [2]: one developed in our laboratory and another from MAKERBOT. Also we studied the quality of printed camera base plates considering two different printing modes which were generated from different orientations with respect to the 3D printer's build plate.

The printed camera base plate was generated horizontally and vertically. In figure 3 is presented the vertically oriented model of the workpiece printed on our printer.

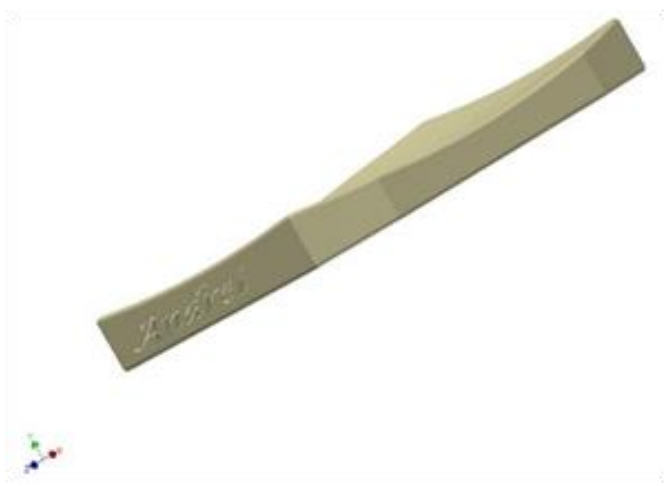

Figure 2: The design of the camera base - Inventor 2016

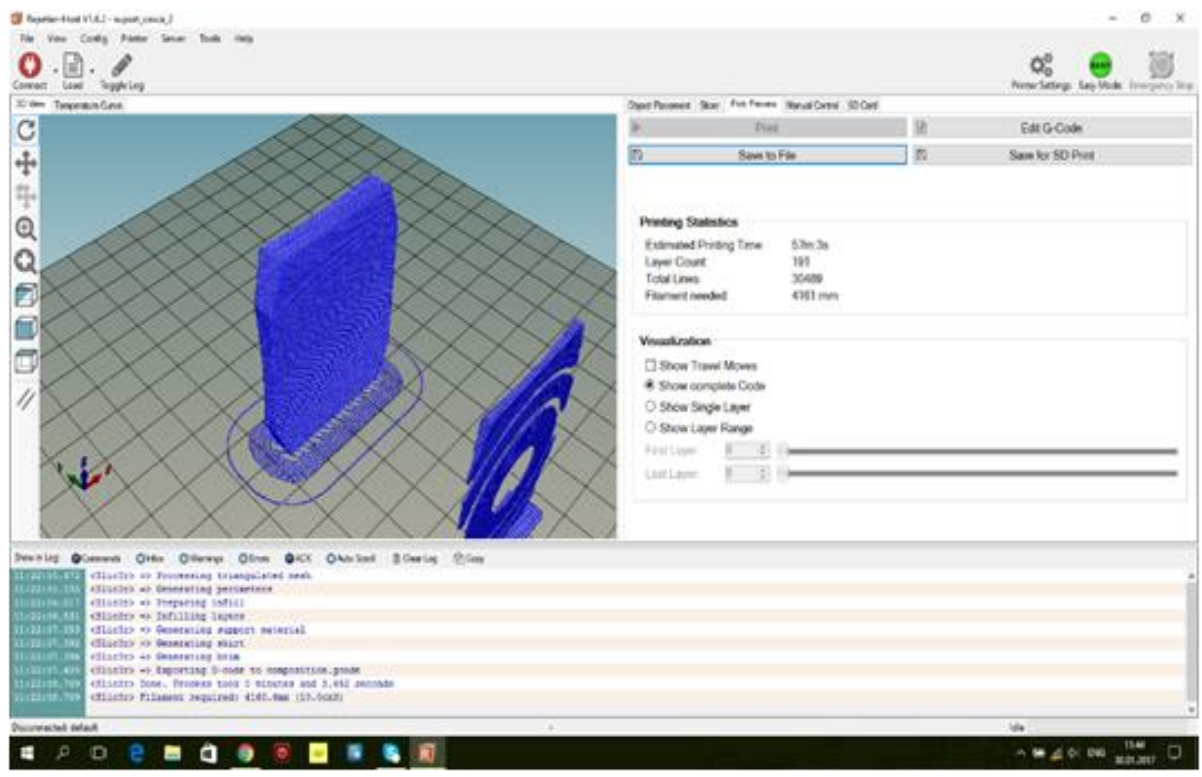

Figure 3: The vertically model on our printer 
In figure 4 is presented the model on horizontal position from MAKERBOT printer. In figure 5 is presented the beginning of the printing process on Makerbot.

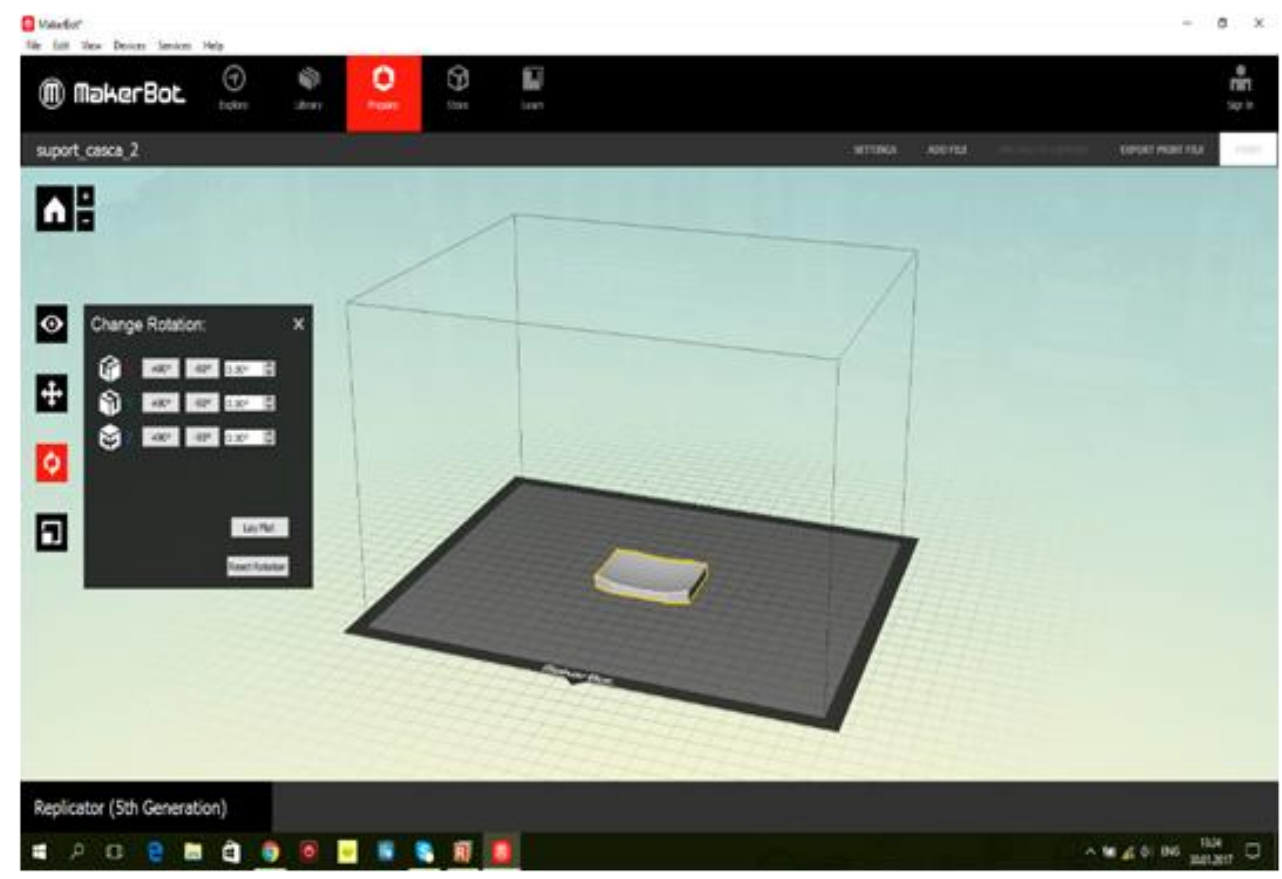

Figure. 4: The horizontally model on MAKERBOT printer

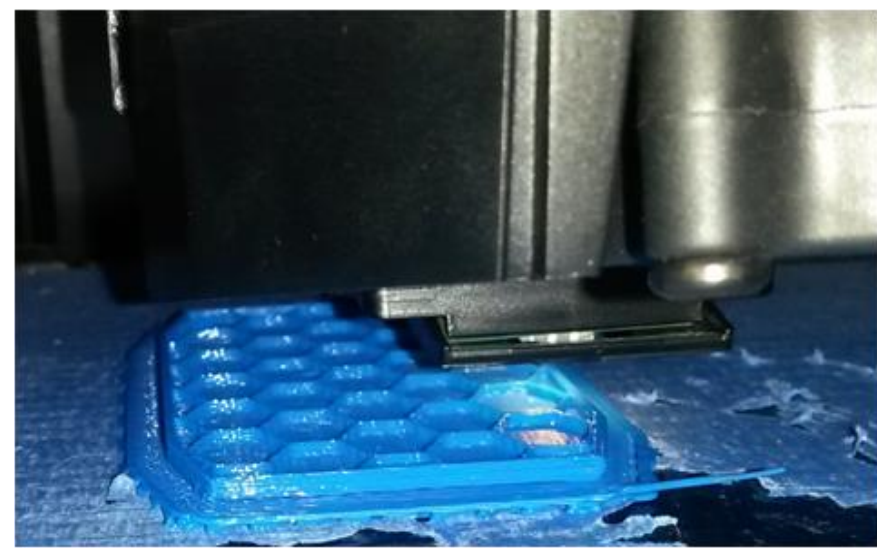

Figure 5: Printed camera base on MAKERBOT printer

If someone open a model in the Prepare section of MakerBot Desktop it will appear on a virtual representation of MakerBot 3D printer's build plate. If the build plate on the screen isn't the right size, then go to Devices > Select Type of Device and select the type of MakerBot 3D printer you're using, [3].

For some parts that have a big upside-down hollow, it is impossible to print in a normal way, the middle roof can fall down during printing process. In this situation, we need to build some supports for them.

Supports are thin layers of plastic web around the object to protect it and support it. Supports are generally not needed if printing small objects.
However, it is really difficult to remove all the supports after printing, see figures 6 and 7 .

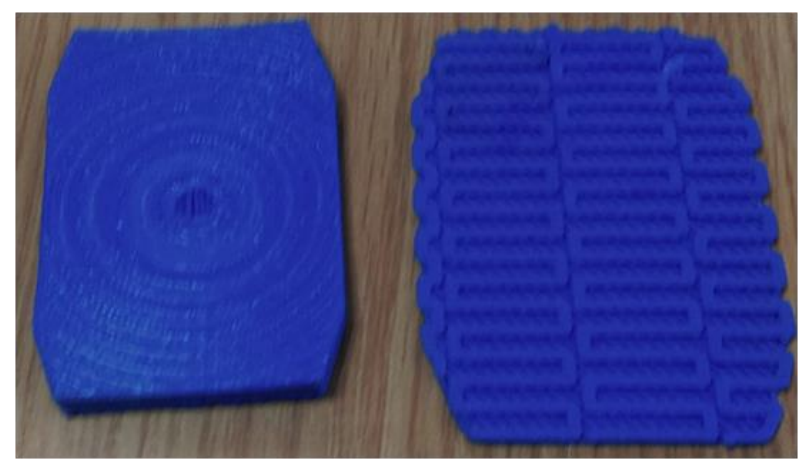

Figure 6: Printed camera base (left) on MAKERBOT printer with built support (right). 


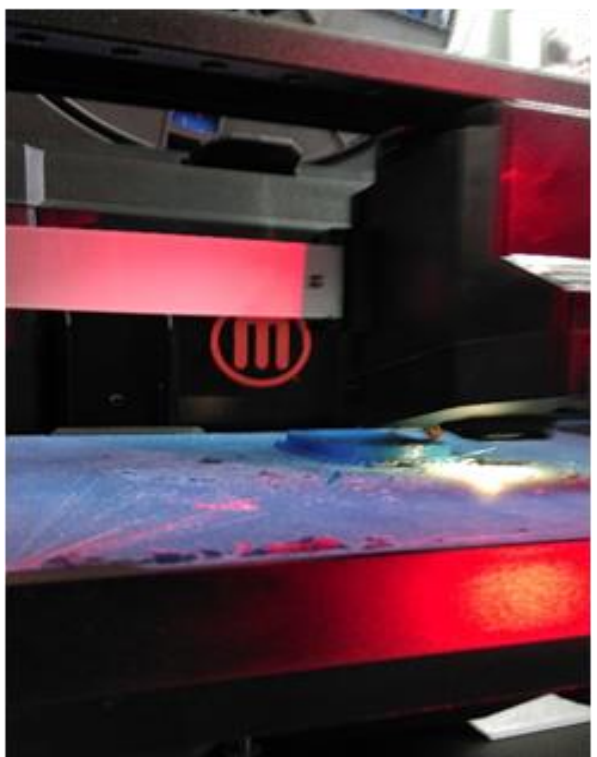

Figure 7: Printing camera base on MAKERBOT

MakerBot 3D printer has become very popular nowadays in many universities and even in some high schools as it is quite easy to setup and use in general.

In figure 8 there is presented our 3D printers with two extruders and two different materials (colours).

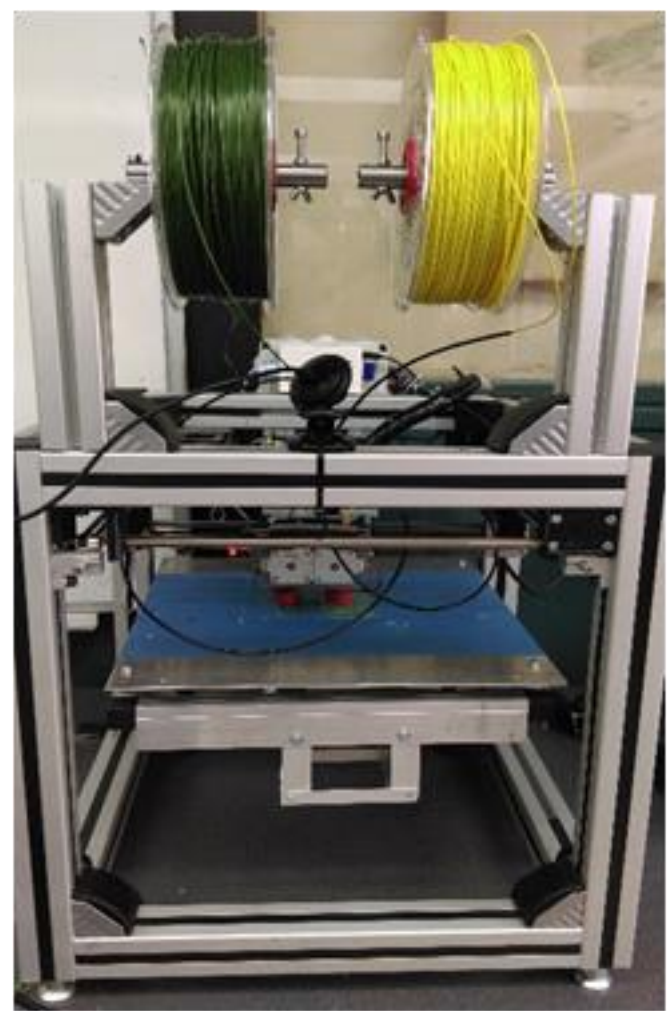

Figure 8: Printing camera base on our 3D Printer

\section{Printing Results}

For most 3D printers, the optimal surface quality is achieved if the surface normal is perpendicular to the printing direction since in this case the relatively high $\mathrm{x} / \mathrm{y}$-resolution plus the physical smoothing effect emerging, e.g., from the melted plastic in fused deposition can be exploited.

The more the surface normal aligns with the (positive or negative) printing direction the more staircase artefacts appear between layers. Because as the surface normal tilts towards the negative printing direction ("overhang"), auxiliary support structures (build support) have to be generated so that the printing quality in surface region touching the support structures will be poor and the removal of support structures may cause additional damage to the object's surface.

In figure 9, the printed camera bases are placed in the printing position. The left one, was printed in a horizontal position, using one material (blue colour) on MAKERBOT printer.

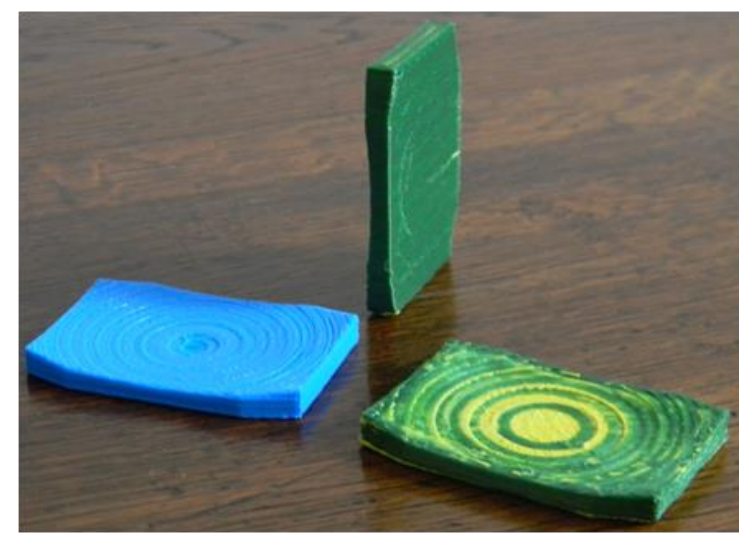

Figure 9: Three printed camera bases from different materials

The centre piece was printed in a vertical position using two materials (green and yellow) and our printer with two extruders.

The piece placed in the right was printed in horizontal position, using two different materials. All build supports were removed. The build supports are larger for horizontal printing and smaller for vertical printing. Printing times are somewhat closed, but there are differences about 10-20 minutes.

Printing with two extruders is slower than with one extruder. The differences are about 30-40 minutes. About surface quality: a smooth surface is obtained for horizontal printing on upper and lower surfaces but a poor quality on borders (side surfaces). 
For vertical printing the smooth surfaces are placed on side surfaces and instead the upper and lower surfaces are of a poor quality. If there are used different materials on a layer, the surface quality is poorer than using the same material on a layer.

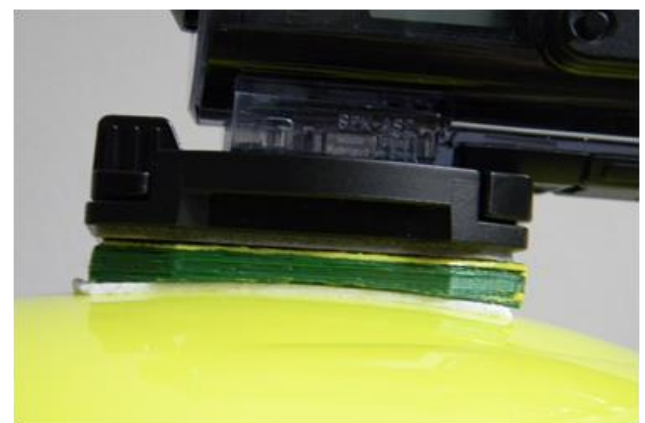

Figure 10: The helmet with printed camera base and action camera

\section{Conclusions}

By using Autodesk Inventor with Makerbot 3D printer, we have created a prototype in a fastest way. Also we used another printer, developed in our laboratory.

This printer has two extruders. Using two different 3D printers and two different materials (colours) we were able to compare surface quality, printing times, build supports of the workpieces.

For each printer the workpieces were printed in horizontal and vertical positions.
For the 3D printer with two extruders there were printed the workpieces on horizontal and vertical positions using two different material colours.

Using two different materials the surface quality is better for the vertical printed position.

\section{References}

[1] Spanu, A., Dontu, O., Besnea, D., Avram, M.: Improvements on Design of Proppeller for Chemical Compound Mixer, Revista de Chimie Volume 66, ISSUE 3; March (2015).

[2] Besnea, D., Avram, M., Constantin, V., Spanu, A., Chivu, L.: Additive Micromanufacturing Technologies for Composite Materials based on Nanotubes and Polymers, The Romanian Review Precision Mechanics, Optics \&Mechatronics, No. 50/(2016).

[3] Cioboata, D., Dontu, O., Besnea, D., Ciobanu, R., Soare, A.: Mechatronic Equipment for Bearing Ring Surface Inspection, The Romanian Review Precision Precision Mechanics, Optics \&Mechatronics, No. 48, (2015).

[4] Berce, P., Balc, N., a.o.: Tehnologii de fabricatie prin adaugare de material si aplicatiile lor, Editura Academiei Romane, Bucuresti(2014).

[5] Prisăcaru,Gh.,Bercea, M., Gramescu, B., Ciupe, V.:Mecatronica aplicata, Ed. Academiei Oamenilor de Stiinta din Romania, București, ISBN 978-6068371-43-6(2011). 